\title{
Pacientes Clínicos Referenciados, mas não Internados na Unidade de Terapia Intensiva: Prevalência, Características Clínicas e Prognóstico*
}

\author{
Referred Medical Patients not admitted to the Intensive Care Unit: \\ Prevalence, Clinical Characteristics and Prognosis.
}

José Rodolfo Rocco ${ }^{1}$, Márcio Soares², Maria de Fátima Gago ${ }^{3}$

\section{RESUMO}

JUSTIFICATIVA E OBJETIVOS: A evolução dos pacientes cuja internação é negada nos centros de terapia intensiva (CTI) é pouco conhecida. Os objetivos deste estudo foram comparar as características dos pacientes internados com aqueles que não foram internados em CTI e identificar os fatores associados com o processo de triagem para a internação.

MÉTODO: Foi realizado um estudo do tipo coorte

1. Médico do Centro de Tratamento Intensivo do Hospital Universitário Clementino Fraga Filho da UFRJ, Professor Adjunto do Departamento de Clínica Médica da Faculdade de Medicina da UFRJ, Mestre e Doutor em Clínica Médica pela UFRJ, Título de Especialista em Terapia Intensiva pela Associação de Medicina Intensiva Brasileira e pela Federação Pan Americana e Ibérica de Medicina e Cuidados Intensivos, Titular-Colaborador do Colégio Brasileiro de Cirurgiões.

2. Médico do Centro de Tratamento Intensivo do Hospital I do Instituto Nacional de Câncer (INCA), Mestre e Doutor em Clínica Médica pela Universidade Federal do Rio de Janeiro, Título de Especialista em Terapia Intensiva pela Associação de Medicina Intensiva Brasileira, Prof. Adjunto do Programa de Pós-Graduação em Ciências do Movimento Humano da UNIGRANRIO, Rio de Janeiro, RJ.

3. Residente de Terapia Intensiva do Hospital Universitário Clementino Fraga Filho da UFRJ. Médica da Rotina do Centro de Tratamento Intensivo da Casa de Saúde São José - Rio de Janeiro.

*Recebido do Centro de Tratamento Intensivo do Hospital Universitário Clementino Fraga Filho da Universidade Federal do Rio de Janeiro, (HUCFF-UFRJ), Rio de Janeiro, RJ

Apresentado em 09 de março de 2006

Aceito para publicação em 01 de junho de 2006

Endereço para correspondência:

Dr. José Rodolfo Rocco

Rua Desenhista Luiz Guimarães, 70 Bloco 1 Apto 602

Barra da Tijuca

22793-261 Rio de Janeiro, RJ

Fone (21) 2431-7301 - Fax. (21) 3325-4579

E-mail: jrrocco@globo.com

(C)Associação de Medicina Intensiva Brasileira, 2006 prospectiva e observacional durante 26 meses. Os dados dos pacientes foram coletados através de um formulário padronizado para a solicitação de internação no CTI. Os desfechos de interesse do estudo foram a internação no CTI e a evolução hospitalar.

RESULTADOS: Foram estudados 455 pacientes dos quais 254 (56\%) foram internados e 201 (44\%) não; a maioria destes, por falta de vagas (82\%). Os pacientes não internados apresentaram maior letalidade $(85 \%$ versus $61 \% ; p<0,001)$. Na análise multivariada, foram identificados os seguintes fatores associados à não internação dos pacientes no CTI [razão de chances, (intervalo de confiança 95\%)]: neoplasia metastática $[5,6(1,7-18,7)]$, pressão arterial sistólica $<90 \mathrm{mmHg}$ $[5,2(3,0-8,8)]$, idade $>70$ anos $[4,0(2,4-6,5)]$, cirrose hepática $[3,7(1,8-7,5)]$, e escala de coma de Glasgow $\leq 5$ [3,6(1,9-6,9)]. Por outro lado, os pacientes em ventilação mecânica $[0,5(0,3-0,7)]$ e aqueles com cardiopatia isquêmica $[0,1(0,03-0,6)]$ apresentaram maior probabilidade de internação no CTI.

CONCLUSÕES: A recusa de pacientes no CTI é freqüente e ocorre geralmente por falta de vagas. Os pacientes não internados apresentaram maior letalidade. Foram identificadas características dos pacientes que estão associadas ao processo de seleção de pacientes para internação na unidade de terapia intensiva.

Unitermos: APACHE II, Critérios de internação, prognóstico, terapia intensiva

\section{SUMMARY}

BACKGROUND AND OBJECTIVES: Information on the outcomes of patients who were refused to the ICU is limited. The aims of this study were to compare the clinical characteristics of patients who were admitted with those of patients who were refused to the ICU and to identify clinical parameters associated with triage 


\section{procedures.}

METHODS - Observational prospective cohort study. The following data were collected using a standard questionnaire: comorbidities, acute illness, vital status, laboratory data and APACHE II score. The end-points of interest were admission to the ICU and vital status at hospital discharge.

RESULTS: A total of 455 patients were studied; 254 (56\%) were admitted and 201 (44\%) were not. The main reason for the refuse of admission was the lack of ICU beds (82\%). Patients who were not admitted had a higher mortality $(85 \%$ vs. $61 \%$; $<<0.001)$. In multivariable analysis, the following variables were associated to non-admission [odds ratio, $(95 \%$ confidence interval)]: metastatic cancer [5.6(1.7-18.7)], arterial systolic pressure $<90 \mathrm{mmHg}[5.2(3.0-8.8)]$, age $>70$ years [4.0(2.4-6.5)], hepatic cirrhosis [3.7(1.8-7.6)], and Glasgow coma scale $\leq 5$ [3.6(1.9-6.9)]. The variables associated with ICU admission were: mechanical ventilation [0.5(0.3-0.7)] and acute coronary syndromes [0.1(0.03-0.6)].

CONCLUSIONS: Refusal of ICU admission is frequent and generally as a consequence of ICU beds shortage. Patients who were not admitted had a higher mortality. Clinical characteristics associated with the refusal of admission were identified suggesting that they are used in clinical decision-making for ICU triage.

Key Words: Admission criteria, APACHE II, intensive care, outcome

\section{INTRODUÇÃO}

O estudo foi aprovado pelo Comitê Institucional de Ética em pesquisa do HUCFF-UFRJ, sendo dispensada a assinatura do termo de consentimento livre e esclarecido.

A falta de leitos para terapia intensiva é comum em diversos países europeus ${ }^{1}$ e, até nos Estados Unidos da América, onde o número de leitos é maior ${ }^{2}$. Similarmente, o Brasil apresenta poucos leitos de terapia intensiva disponíveis para atender toda a demanda de pacientes. Por outro lado, os leitos dos centros de tratamento intensivo (CTI) deveriam ser ocupados por pacientes com probabilidades potenciais de recuperação ${ }^{3}$. Assim, a presença de um leito disponível não deve ser empregada como único critério para a internação no CTI.

A Sociedade Americana de Terapia Intensiva (SCCM) elaborou critérios para admissão e alta da terapia intensiva ${ }^{3}$. O refinamento do processo de triagem para a internação é fundamental para a melhoria do cuidados dos pacientes e da alocação de recursos.

Os objetivos do presente estudo foram avaliar e comparar as características e o prognóstico dos pacientes internados com aqueles recusados na terapia intensiva em um hospital universitário; e, identificar fatores associados com processo de triagem para a internação no CTI.

\section{MÉTODO}

Entre agosto de 2002 e setembro de 2004, foi realizado um estudo prospectivo de coorte no CTI do Hospital Universitário Clementino Fraga Filho (HUCFF) da Universidade Federal do Rio de Janeiro (UFRJ). O HUCFF é um hospital público terciário com 450 leitos e desenvolve um ativo programa de transplante de órgãos. O CTI é constituído por duas unidades de seis leitos: uma reservada para pacientes em pós-operatórios e outra para pacientes clínicos. Duas enfermeiras, quatro técnicos de enfermagem e um fisioterapeuta trabalham em cada unidade. A relação entre os profissionais de enfermagem/paciente oscila entre um e dois. No período da manhã, dois médicos, dois residentes de terapia intensiva e um interno-bolsista estão presentes em cada unidade. Nos períodos da tarde e noite, pelo menos dois médicos e dois interno-bolsistas trabalham em regime de plantão. O CTI possui próteses ventilatórias microprocessadas, monitorização hemodinâmica invasiva e não-invasiva, hemodiálise, endoscopias respiratória e digestiva que podem ser oferecidas para todos os pacientes nas 24 horas.

No período do estudo, todos os pacientes com uma complicação clínica grave, em que foi solicitada uma avaliação quanto ao mérito da internação no CTI foram incluídos. Os pacientes em pós-operatório de cirurgias eletivas foram excluídos, pois a não internação deles por falta de vagas no CTI não traria tanto transtorno quanto para os pacientes clínicos. Nesse caso, a cirurgia geralmente era postergada. Também foram excluídos os menores de 18 anos, as grávidas e puérperas. Os pacientes foram divididos em dois grupos: 1) internados e 2) recusados no CTI. No momento da avaliação do paciente, os seguintes dados foram coletados através de formulários padronizados próprio para o pedido de internação no CTI: idade, sexo, presença de comorbidades, tais como doença cardíaca isquêmica, cirrose hepática, insuficiência renal crônica, insuficiência cardíaca congestiva, doença pulmonar obstrutiva crônica, síndrome da imunodeficiência adquirida, ne- 
oplasias hematológicas (linfomas, mieloma múltiplo ou leucemias), neoplasia metastática e imunossupressão, doenças agudas [insuficiência renal aguda, hemorragia digestiva alta, parada cardiorrespiratória, coma (escala de coma de Glasgow $\leq 5$ ), pressão arterial sistólica < $90 \mathrm{mmHg}$, presença de infecção, ventilação mecânica e uso de vasopressores (exceto dopamina em doses $<5 \mu \mathrm{g} / \mathrm{kg} / \mathrm{min}$ )], sinais vitais (pressão arterial, freqüências cardíaca e respiratória, temperatura axilar, escala de coma de Glasgow) e exames de laboratório (hematócrito, contagem de leucócitos, creatinina, sódio e potássio séricos), gasometria arterial e fração de oxigênio inspirado. O escore APACHE II ${ }^{4}$ Acute Physiology and Chronic Health Evaluation, número de pontos e probabilidade percentual de óbito, foi calculado para todos os pacientes. Normalmente, para o cálculo deste escore, são considerados os piores dados (exames de laboratório e dados de exame clínico / sinais vitais mais alterados) nas primeiras 24 horas após a admissão na UTI. Nos pacientes não internados, decidiu-se coletar os dados na hora do pedido da admissão no CTI, em concordância com dados da literatura ${ }^{7}$. Para efeito do cálculo do escore APACHE II, os eventuais dados não coletados foram considerados normais ${ }^{4}$. 0 estudo não interferiu com as condutas e as decisões clínicas relacionadas aos pacientes. A internação no CTI e a evolução hospitalar foram os desfechos de interesse do estudo. É importante assinalar que durante a vigência do estudo não existia nenhuma diretriz institucional quanto ao preenchimento das vagas do CTI. A avaliação quanto ao mérito da internação no CTI era feita pelos médicos da rotina ou do plantão com base em seus conhecimentos médicos e na disponibilidade de leito

Os dados categóricos foram comparados pelos testes Qui-quadrado (com correção de Yates quando indicado) ou Exato de Fisher; os dados contínuos foram avaliados pelo Mann-Whitney $\mathrm{U}$, pois as variáveis não apresentavam distribuição normal. As variáveis categóricas foram apresentadas como percentuais e as variáveis contínuas, como valores de mediana (intervalo interquartil 25\%-75\%). A regressão logística binária foi utilizada para avaliar a contribuição independente das covariáveis na probabilidade de recusa da internação no CTI. Para isso, as variáveis com um valor de $p<0,2$ na avaliação univariada e aquelas consideradas clinicamente relevantes entraram na análise multivariada. Antes da realização da análise multivariada, foram estudadas as possíveis interações entre variáveis. Quando existiu interação, selecionou-se aquela com maior poder estatístico. O escore APACHE II não foi incluído como co-variável, pois diversas variáveis presentes no estudo são utilizadas para o cálculo do escore. As variáveis contínuas foram estudadas individualmente para determinação dos melhores pontos de corte para a transformação em variáveis categóricas. $\mathrm{Na}$ avaliação da discriminação, foi utilizado o cálculo da área sob a curva receiver operating characteristic (ROC) $)^{5}$. A calibração foi avaliada com o teste do goodnessof-fit (GOF) de Hosmer-Lemeshow ${ }^{6}$. Nessas análises foram utilizados os programas Sigma Stat 3.0 (SPSS Inc., Chicago, IL, EUA) e SPSS versão 10.0 (SPSS Inc., Chicago, IL, EUA). O valor de $p<0,05$ foi considerado estatisticamente significativo.

\section{RESULTADOS}

\section{Caracterização da População Estudada}

Foram estudados 455 pacientes dos quais 254 (56\%) foram internados e 201 (44\%) não o foram. Na tabela 1 , são enunciadas e comparadas as características e a evolução dos pacientes. Na tabela 2, são comparados os sinais vitais e os exames complementares dos dois grupos. Nestas análises, foram observadas diferenças significativas entre os dois grupos. Os pacientes internados no CTI apresentaram mais freqüentemente cardiopatia isquêmica, infecção, hematócrito mais elevado e maior número de leucócitos. Já aqueles que não foram internados apresentaram idade mais elevada, maiores prevalências de cirrose hepática, neoplasia metastática, imunodepressão, coma (escala de coma de Glasgow $\leq 5)$, pressão arterial sistólica e diastólica mais baixas, uso mais freqüente de vasopressores e freqüência cardíaca e temperatura axilar mais elevadas. A probabilidade de óbito do escore prognóstico APACHE II foi mais elevada nos pacientes não internados (mas não o número de pontos), assim como o percentual de óbitos. Tal fato pode ser atribuído a uma maior incidência de sepse nos pacientes não admitidos, cujo coeficiente utilizado para o cálculo da probabilidade de óbito é mais elevado.

Os serviços hospitalares nos quais os pacientes não internados estavam internados quando do pedido de admissão no CTI foram diversos, sendo a maioria do Serviço de Clínica Médica (60\%), Enfermaria de Cuidados Intensivos (unidade intermediária pós-operatória) (11\%), Doenças Infecciosas e Parasitárias (7\%), Pneumologia (6\%), Emergência (5\%), Hematologia (4\%) e outras (7\%). 
PACIENTES CLÍNICOS REFERENCIADOS, MAS NÃO INTERNADOS NA UNIDADE DE

TERAPIA INTENSIVA: PREVALÊNCIA, CARACTERÍSTICAS CLÍNICAS E PROGNÓSTICO

Tabela 1 - Características Epidemiológicas, Comorbidades, Doenças Agudas, Escore Prognóstico APACHE II e Evolução dos Pacientes Internados e não Internados no CTI.

\begin{tabular}{|c|c|c|c|}
\hline Características & Pacientes Internados $(n=254)$ & Pacientes Não Internados ( $\mathrm{n}=201)$ & $\mathrm{p}$-valor \\
\hline Idade (anos) & $52(31-66)$ & $63(47-75)$ & $<0,01$ \\
\hline Cardiopatia isquêmica & $13 / 254-5,1 \%$ & $2 / 201-1,0 \%$ & 0,03 \\
\hline Cirrose hepática & $15 / 254-5,9 \%$ & $36 / 201-17,9 \%$ & $<0,01$ \\
\hline Insuficiência renal crônica & $28 / 254-11,0 \%$ & $19 / 201-9,5 \%$ & 1,0 \\
\hline Insuficiência cardíaca congestiva & $20 / 254-7,9 \%$ & $24 / 201-11,9 \%$ & 0,2 \\
\hline DPOC & $27 / 254-10,6 \%$ & $28 / 201-13,9 \%$ & 0,4 \\
\hline SIDA & $20 / 254-7,9 \%$ & $18 / 201-9,0 \%$ & 0,8 \\
\hline Neoplasia hematológica & $16 / 254-6,3 \%$ & $10 / 201-5,0 \%$ & 0,7 \\
\hline Neoplasia metastática & $4 / 254-1,6 \%$ & $19 / 201-9,5 \%$ & $<0,01$ \\
\hline Imunodepressão & $5 / 254-2,0 \%$ & $21 / 201-10,4 \%$ & $<0,01$ \\
\hline Insuficiência renal aguda & $49 / 254-19,3 \%$ & $37 / 201-18,4 \%$ & 0,9 \\
\hline Hemorragia digestiva & $15 / 254-5,9 \%$ & $18 / 201-9,0 \%$ & 0,3 \\
\hline Parada cardiorrespiratória* & $17 / 254-6,7 \%$ & $6 / 201-3,0 \%$ & 0,1 \\
\hline Escala de coma de Glasgow $\leq 5$ & $20 / 254-7,9 \%$ & $39 / 201-19,4 \%$ & $<0,01$ \\
\hline PAS < 90 mmHg à admissão & $30 / 254-11,8 \%$ & $81 / 254-40,3 \%$ & $<0,01$ \\
\hline Presença de infecção & $151 / 254-59,4 \%$ & $105 / 201-52,2 \%$ & 0,04 \\
\hline Ventilação mecânica & $192 / 254-75,6 \%$ & $138 / 201-68,7 \%$ & 0,1 \\
\hline Uso de vasopressores & $89 / 254-35,0 \%$ & $98 / 201-48,8 \%$ & $<0,01$ \\
\hline APACHE II - pontos & $19(14-26)$ & $17,5(13-23)$ & 0,08 \\
\hline APACHE II (\%) & $26,3(12,9-46,4)$ & $32,6(20,2-56,2)$ & 0,01 \\
\hline Óbito hospitalar & $156 / 254-61,4 \%$ & $171 / 201-85,1 \%$ & $<0,01$ \\
\hline
\end{tabular}

*antes da internação no CTI ou antes do pedido de internação;

DPOC - doença pulmonar obstrutiva crônica; SIDA - síndrome da imunodeficiência adquirida; PAS - pressão arterial sistólica; APACHE - Acute Physiology and Chronic Health Evaluation.

Tabela 2 - Sinais Vitais e Exames de Laboratório dos Pacientes Internados e dos não Internados no CTI.

\begin{tabular}{|c|c|c|c|}
\hline & Pacientes Internados $(n=254)$ & Pacientes não Internados $(\mathrm{n}=201)$ & $\mathrm{p}$-valor \\
\hline PAS (mmHg) & $96(81-154)$ & $110(90-130)$ & 0,1 \\
\hline PAD (mmHg) & $60(47-94)$ & $70(60-80)$ & 0,2 \\
\hline Freqüência cardíaca (bpm) & $100(85,8-115)$ & $111(68-132)$ & $<0,01$ \\
\hline Freqüência respiratória (irpm) & $24(21-30)$ & $24(18-28)$ & $<0,01$ \\
\hline Temperatura axilar $\left({ }^{\circ} \mathrm{C}\right)$ & $36,5(35,5-37,9)$ & $37(37-37)$ & $<0,01$ \\
\hline Escala de coma de Glasgow & $15(12-15)$ & $15(9-15)$ & 0,03 \\
\hline Hematócrito (\%) & $30(25,2-35)$ & $21(27,6-36)$ & 0,05 \\
\hline $\mathrm{N}^{\circ}$ leucócitos $/ \mathrm{mm}^{3}$ & $12250(8200-17500)$ & $10900(7675-14825)$ & 0,02 \\
\hline $\mathrm{pH}$ & $7,39(7,26-7,49)$ & $7,39(7,25-7,46)$ & 0,8 \\
\hline $\mathrm{PaO}_{2}(\mathrm{mmHg})$ & $114,1(79,5-172,9)$ & $102,4(78,1-175,5)$ & 0,4 \\
\hline $\mathrm{FiO}_{2}(\%)$ & $50(36-100)$ & $40(36-100)$ & 0,2 \\
\hline Relação $\mathrm{PaO}_{2} / \mathrm{FiO}_{2}$ & $246,0(150,0-383,2)$ & $253,0(156,8-375,8)$ & 0,6 \\
\hline Creatinina (mg/dL) & $1,2(0,7-2,3)$ & $1,4(0,8-2,45)$ & 0,1 \\
\hline Sódio (mEq/L) & $138(134-143)$ & $138(133-142)$ & 0,3 \\
\hline Potássio (mEq/L) & $4,15(3,6-4,9)$ & $4,3(3,7-4,9)$ & 0,3 \\
\hline
\end{tabular}

PAS - pressão arterial sistólica; PAD - pressão arterial diastólica; $\mathrm{FiO}_{2}$ - fração de oxigênio inspirado.

Evolução dos Pacientes durante a Internação Hospitalar

Os pacientes não admitidos apresentaram uma letalidade maior do que os internados no CTI $(85 \%$ versus $61 \% ; \mathrm{p}<0,001)$. A taxa de mortalidade padronizada (TMP) (óbitos observados/óbitos preditos pelo escore APACHE II) foi elevada e também revelou diferenças entre os pacientes internados [TMP $=1,80$ (intervalo de confiança de 95\% (IC95\%) = 1,65-1,99)] e aqueles não internados: [TMP $=2,21($ IC95\% $=2,02-2,40)]$.

Foram observadas duas grandes causas para a não admissão pacientes no CTI: a ausência de indicação - 36/201 (18\%) e a ausência de leito disponível - 165/201 (82\%). A maior parte dos pacientes sem indicação estava "muito bem" para ser internada $(n=$ 27 ), enquanto que outros pacientes foram considera- 
dos muito graves para terem esse benefício $(n=9)$. A letalidade hospitalar desses dois grupos de pacientes foi significativamente diferente: pacientes muito bem - 44\% (12/27) versus pacientes muito graves - 89\% (8/9); $p=0,026$. Os pacientes sem indicação para a internação no CTI apresentaram menor letalidade hospitalar em relação aos pacientes recusados por falta de vagas [21/36 (58,3\%) versus 151/165 (91,5\%), $p<$ $0,0001]$. Entretanto, o escore APACHE II em pontos (18 $\pm 8,2$ versus $20,1 \pm 8,3, p=0,19$ ) e a probabilidade de óbito $(29,2 \%$ (16,1-47,1\%) versus $34,8 \%(20,4-56,8 \%)$, $p=0,21)$ foram semelhantes entre os dois grupos.

\section{Fatores Associados com a Recusa da Internação}

Os resultados da análise multivariada dos fatores associados à recusa da internação no CTI estão apresentados na tabela 3. Foram positivamente associados, ou seja, com maior probabilidade de serem recusados, os pacientes com as seguintes características: neoplasia metastática, PAS $<90 \mathrm{mmHg}$ à admissão, idade $>70$ anos, cirrose hepática e escala de coma de Glasgow $\leq$ 5. Todos com razão de probabilidade elevada (acima de 3). Por outro lado, os pacientes em ventilação mecânica e aqueles com cardiopatia isquêmica tiveram maior probabilidade de serem internados.

Tabela 3 - Análise Multivariada dos Fatores Associados à não Internação no CTI ( $\mathrm{n}=455)$.

\begin{tabular}{lcc}
\hline Variáveis & $\begin{array}{c}\text { Razão de } \\
\text { Probabilidades } \\
(\text { IC 95\%) }\end{array}$ & p-valor \\
\hline Neoplasia metastática & $5,626(1,690-18,733)$ & 0,005 \\
PAS $<90$ mmHg à admissão & $5,182(3,041-8,831)$ & $<0,001$ \\
Idade $>70$ anos & $3,977(2,418-6,540)$ & $<0,001$ \\
Cirrose hepática & $3,737(1,851-7,545)$ & $<0,001$ \\
Escala de coma de Glasgow $\leq 5$ & $3,612(1,882-6,933)$ & $<0,001$ \\
Ventilação mecânica & $0,455(0,280-0,741)$ & 0,002 \\
Cardiopatia isquêmica & $0,126(0,025-0,629)$ & 0,012 \\
\hline
\end{tabular}

Teste do goodness-of-fit (GOF) de Hosmer-Lemeshow =3,023; $p=0,883$. Area sobre a curva ROC $=0,798($ IC95\% $=0,756-0,839)$

IC 95\% - intervalo de confiança a 95\%; PAS - pressão arterial sistólica.

\section{DISCUSSÃO}

No presente estudo, $44,2 \%$ dos pacientes avaliados para internação no CTI foram recusados. Elevadas taxas de recusa nas internações têm sido relatadas ${ }^{7-12}$. Quando os resultados destes estudos são avaliados em conjunto, de um total de 3650 pacientes, a proporção de recusa variou de $16 \%$ a $51,2 \%$ com média de $29,4 \% \pm 12,9 \%$. Em estudo que incluiu apenas pacientes cirúrgicos ${ }^{11}$, a freqüência de recusa foi bem menor, de apenas 4,2\%. O valor do escore APACHE II foi semelhante entre os pacientes internados $(12,8$ \pm 1 pontos) e recusados (14,9 $\pm 1,3$ pontos) em dois estudos $^{7-12}$. Também não foram observadas diferenças significativas entre os pacientes admitidos e aqueles não admitidos. Entretanto, o valor do escore observado no presente estudo foi mais elevado (admitidos 19 pontos; não admitidos - 17,5 pontos). Confirmando essa observação, os pacientes não admitidos nesses estudos apresentaram sempre idade superior àqueles internados ${ }^{7-12}$.

Nos estudos em que a letalidade foi anotada ${ }^{7,9,11,12}$, esta foi maior nos pacientes recusados. Nesses estudos, a letalidade média dos pacientes admitidos foi de $26 \pm$ $14,8 \%$, enquanto que a daqueles não admitidos foi de $48,8 \% \pm 9,5 \%$. No presente estudo, a diferença foi menor, pois a letalidade observada foi menos elevada nos internados $(61,4 \%)$ do que nos recusados $(85,1 \%)$.

Em estudo prospectivo realizado em $26 \mathrm{CTI}$ franceses ${ }^{8}$, os pacientes não internados apresentaram idade mais elevada (> 65 anos) e mau estado de saúde crônica. Nesse estudo, os principais diagnósticos dos pacientes admitidos foram: insuficiência respiratória, insuficiência renal, choque e coma. Por outro lado, doenças crônicas respiratórias e cardíacas ou doença metastática sem perspectiva de remissão foram associadas com a recusa à admissão. Tais dados são muito semeIhantes aos deste estudo, em relação a não admissão de pacientes idosos (> 70 anos), portadores de doenças crônicas (cirrose hepática) e neoplasia metastática e à admissão de pacientes sob ventilação mecânica. Entretanto, contrariamente, observou-se que pacientes em coma (escala de Glasgow $\leq 5$ ) e com choque (PAS $<90 \mathrm{mmHg}$ ) apresentaram menor probabilidade de internação no CTI.

Em estudo realizado em Hong Kong ${ }^{9}$, as razões para a recusa foram: triagem $(n=104)$, futilidade $(n=82)$ e internações não apropriadas (pacientes muito bem, $n$ $=50$ ). Através da avaliação da taxa de mortalidade padronizada (TMP), verificaram que os pacientes não internados apresentaram valor mais elevado em relação aos que foram admitidos [TMP $=1,24$ (IC95\% $=1,05$ 1,46) dos não admitidos contra TMP = 0,93 (IC95\% = 0,78-1,09) dos admitidos]. Entretanto, a TMP foi maior nos pacientes com prognóstico intermediário (TMP = 1,95 (IC95\% = 1,19-3,20). A recusa da internação foi mais freqüentemente associada à idade avançada, grupo diagnóstico e gravidade da doença. Os autores concluíram que os pacientes com prognóstico inter- 
mediário apresentaram maior mortalidade e que estratégias deveriam ser criadas para identificar pacientes desse grupo que se beneficiariam da admissão no CTI. Assim, pacientes muito graves ou muito pouco graves não seriam bons candidatos para a terapia intensiva ${ }^{13}$. Assim, o grupo de prognóstico intermediário (ou incerto) seriam os melhores candidatos, pois a terapia intensiva poderia potencialmente modificar o prognóstico. Também se observou que a TMP foi mais elevada nos pacientes não internados, apesar dos valores serem bem mais elevados.

Em outro estudo realizado em 11 hospitais franceses ${ }^{10}$ as razões para a recusa foram: "muito bem" para beneficiar-se $(55,4 \%)$, muito enfermo para beneficiar-se $(37,2 \%)$, unidade muito atarefada $(6,5 \%)$ e recusa pela família $(0,7 \%)$. Através da regressão logística, foram identificados os seguintes fatores relacionados com a recusa da internação na terapia intensiva: dependência de cuidados, câncer metastático, unidade sem vaga e hospital estudado.

No presente estudo verificou que pacientes em ventilação mecânica e aqueles portadores de cardiopatia isquêmica apresentaram maior probabilidade de serem internados no CTI. A principal causa de solicitação de internação no CTI é a insuficiência respiratória. Logo, não surpreende que a necessidade de ventilação mecânica esteja associada à maior probabilidade do paciente ser internado no CTI. A maioria dos pacientes com angina pectoris ou infarto agudo do miocárdio apresentaram melhor prognóstico e boa qualidade de vida após o episódio, desde que tratado precocemente. Este fato também foi observado em outro estudo ${ }^{15}$. Em estudo realizado em cinco hospitais israelenses ${ }^{1}$, os pesquisadores observaram que a sobrevida foi maior nos pacientes internados na terapia intensiva nos três primeiros dias seguintes à deterioração do quadro clínico. Essa melhor sobrevida sugere a existência de uma janela de oportunidade crítica para os pacientes beneficiarem-se da terapia intensiva. llustrando esse fato, quanto mais tempo um paciente persiste em choque, maior a probabilidade dele desenvolver disfunção de órgãos e sistemas irreversível ${ }^{16}$. Os autores concluíram que o aumento da rotatividade de pacientes na terapia intensiva, possibilitando que novos pacientes recebam cuidados intensivos mais precocemente pode ser vantajoso. Porém, para proceder essa rotatividade, também é necessário estabelecer critérios objetivos para a alta do paciente do CTI. Altas prematuras principalmente no período noturno, estão associadas à maior letalidade hospitalar ${ }^{17}$ e maiores taxas de re-interna- ção ${ }^{18}$. Idealmente, os hospitais deveriam contar com recursos para cuidar dos pacientes que se encontram em melhores condições clínicas, mas que ainda não se recuperaram totalmente, ou dos pacientes cronicamente ventilados ${ }^{19}$. A solução seria a criação de unidades intermediárias e unidades de desmame, desde que os leitos para terapia intensiva fossem suficientes para a demanda ${ }^{19}$. Por outro lado, existe o risco de que essas unidades possam se transformar em CTI com poucos recursos. Em momentos de restrição dos leitos de terapia intensiva, as cirurgias eletivas deveriam ser suspensas e os pacientes internados na emergência ou em recuperação pós-operatória deveriam continuar em suas respectivas unidades. Também deveriam ser suspensas as internações para monitorização dos pacientes, dando preferência para a internação de pacientes para tratamento ativo.

Uma outra alternativa para a falta de leitos em unidades de terapia intensiva seria o tratamento intensivo fora da UTI (enfermaria ou quarto). Para isso seria necessária uma equipe treinada em consultoria e equipamentos (monitores, próteses ventilatórias, bombas de infusão, etc. $)^{20}$. O objetivo desta intervenção precoce é a prevenção da deterioração posterior do quadro clínico e a melhora do prognóstico do paciente até que ele seja finalmente internado no CTI ${ }^{16,21}$. É interessante notar que no estudo israelense ${ }^{7}$, os pacientes internados tardiamente $(36 \%)$ apresentaram uma letalidade intermediária entre os internados imediatamente (14\%) e aqueles nunca internados (46\%).

Uma opção simplista seria a ampliação dos leitos para a terapia intensiva até o total atendimento da demanda, mesmo em momentos de pico. Assim, não haveria necessidade de triagem de pacientes, pois as vagas estariam prontamente disponíveis. Entretanto, esta estratégia esbarra em alguns riscos. A maior oferta de vagas ocasiona a internação de pacientes pouco graves e sem indicação para monitorização, o que acarretaria um aumento dos custos da internação hospitalar. Além disso, o custo do investimento de se manter um grande CTI vazio (custo material e de pessoal) pode ser proibitivo ${ }^{19}$. Em alguns estudos em que o número de leitos para terapia intensiva foi diminuído ${ }^{15,22,23}$ foi observado que o número de intervenções por paciente aumentou, o número de dias de internação no CTI e hospitalar diminuíram e a proporção de pacientes cirúrgicos e pacientes clínicos não provenientes do serviço de Emergência aumentaram. Em um dos estudos $^{23}$, foi observada diminuição do escore APACHE II. Entretanto, nenhum dos estudos demonstrou aumento 
da letalidade com a diminuição da disponibilidade de leitos. No presente estudo, somando-se os pacientes internados ( $n=254$ ) com aqueles que não foram internados por falta de leitos disponíveis $(n=165)$, calculou-se que para a internação desses 419 pacientes, o CTI deveria ter aproximadamente dez leitos, ao invés dos seis existentes.

Finalmente, deve-se levar em consideração as expectativas do paciente e de seus familiares. Por razões de foros íntimos, social, religioso ou de outra natureza, o paciente e/ou seus familiares podem não desejar a admissão no CTI. O médico deve sempre discutir com o paciente ou seus familiares (quando o paciente não puder se expressar) sobre as preferências em relação à terapia intensiva.

\section{LIMITAÇÕES DO ESTUDO}

O presente estudo foi realizado em um hospital público universitário situado em um grande centro urbano com número de leitos para terapia intensiva claramente insuficiente para a demanda. Foram estudadas apenas as solicitações de pacientes clínicos, comparados com os pacientes clínicos admitidos. Os resultados deste estudo não devem ser generalizados para pacientes cirúrgicos. Da mesma forma que é necessário cautela com a sua extrapolação para outros grupos de pacientes ou para outros hospitais públicos ou privados.

\section{CONCLUSÕES}

O presente estudo revelou que a recusa de pacientes no CTI é comum e que os motivos relacionados incluem idade avançada, presença de doenças crônicas, doenças agudas graves e falta de leitos. Apesar de não existirem critérios objetivos para a triagem de pacientes no CTI-HUCFF/UFRJ, o presente estudo revelou a existência de um processo de seleção de pacientes para a internação na terapia intensiva. Também se observou que aqueles pacientes submetidos à triagem e cuja internação no CTI foi negada apresentaram maior letalidade. São necessários estudos objetivos e ampla discussão multiprofissional com as sociedades médica e civil sobre o tema.

\section{REFERÊNCIAS}

01. Simchen E, Sprung CL, Galai N et al - Survival of critically ill patients hospitalized in and out of intensive care units under paucity of intensive care unit beds. Crit Care Med, 2004;32:1654-1661.

02. Franklin C, Rackow EC, Mandoni B et al - Triage considerations in medical intensive care. Arch Intern Med, 1990;150:1455-1459.

03. Task Force of the American College of Critical Care Medicine. Guidelines for intensive care unit admission, discharge, and triage. Crit Care Med, 1999;27:633-638.

04. Knaus WA, Draper EA, Wagner DP et al - APACHE II: A severity of disease classification. Crit Care Med, 1985;13:818-829.

05. Hanley JA, McNeil BJ - The meaning and use of the area under a receiver operating characteristic (ROC) curve. Radiology, 1982;143:2936.

06. Hosmer DW, Lemeshow S - Applied Logistic Regression. $2^{\text {nd }}$ Ed, New York, Wiley-Interscience, 2000.

07. Sprung CL, Geber, D. Eidelman LA et al - Evaluation of triage decisions for intensive care admission. Crit Care Med, 1999;27:10731079.

08. Azoulay E, Pochard F, Chevret S et al - Compliance with triage to intensive care recommendations. Crit Care Med, 2001;29:2132-2136.

09. Joynt GM, Gomersall CD, Tan P et al - Prospective evaluation of patients refused admission to an intensive care unit: triage, futility and outcome. Intensive Care Med, 2001;27:1441-1445.

10. Orgeas-Garrouste M, Montuclard L, Timsit J-F et al - Predictors of intensive care unit refusal in French intensive care units: A multiplecenter study. Crit Care Med, 2005;33:750-755.

11. Metcalfe MA, Sloggett A, McPherson K - Mortality among appropriately referred patients refused admission to intensive care units. Lancet, 1997;350:7-12.

12. Friso-Lima P, Gurman D, Shapira A et al - Rationing critical care - what happens to patients who are not admitted?. Theoretical Surgery, 1994;39:208-211.

13. Marshall MF, Schwenzer KJ, Orsina et al - Influence of political power, medical provincialism, and economic incentives on the rationing of surgical intensive care unit beds. Crit Care Med, 1992;20:387394.

14. Society of Critical Care Ethics Committee: Consensus statement on the triage of critically ill patients. JAMA 1994; 271:1200-1203.

15. Singer DE, Carr PL, Mulley AG et al - Rationing intensive care - physicians responses to a resource shortage. $\mathrm{N}$ Engl J Med, 1983;309:1155-1160.

16. Higgins TL, McGee WT, Steingrub JS et al - Early indicators of prolonged intensive care unit stay: Impact of illness severity, physician staffing, and pre-intensive care unit length of stay. Crit Care Med, 2003;31:45-51.

17. Goldfrad C, Rowan K - Consequences of discharges from intensive care at night. Lancet, 2000;355:1138-1142.

18. Sinuff T, Kahnamoui K, Cook, DJ et al - Rationing critical care beds: A systematic review. Crit Care Med, 2004;32:1588-1597.

19. Szalados JE - Access to critical care: Medical rationing of a public right or privilege? Crit Care Med, 2004;32:1623-1624.

20. Goldhill DR, McNarry A - Intensive care outreach services. Curr Anaesth Crit Care, 2002;13:356-361.

21. Riley B, Faleiro R - Critical care outreach: rationale and development. Br J Anaesth, 2001;5:146-149.

22. Walther SM, Jonasson U - A prospective cohort study of 6-month mortality in a comunity hospital experiencing a gradual reduction in critical care services. Intensive Care Med, 2001;27:700-705.

23. Byrick RJ, Mazer CD, Caskennette GM - Closure of an intermediate care unit. Impact on critical care utilization. Chest, 1993;104:876881. 